Jacek Makowski

\title{
Zum SPRACHLICHEN AUSDRUCK VON HASS
}

Die Vielschichtigkeit und Komplexität des Phänomens der Sprachverwendung im öffentlichen Raum im weiteren, und im Raum der Politik im engeren Sinn ermöglicht eine Vielzahl möglicher Zugänge und Ansätze. Angesichts der Offenheit und Breite der Diskussion, so Holly (1990: 3), verbietet sich wohl zu Recht selbst der Versuch eines vollständigen Überblicks über die Literatur zur Sprache in der Politik. Doch allein aus einer ansatzweise vorgenommenen Betrachtung des Forschungsstandes zum politischen Sprachgebrauch ergibt sich das Vorhandensein von zwei metaphorischen Feldern, mit denen versucht wird, den öffentlichen, insbesondere den politischen Diskurs zu beschreiben - Krieg und Theater (vgl. Bralczyk 2015, siehe hierzu auch Bralczyk in diesem Band). Die Sprache verschärft und brutalisiert sich vor allem dann, wenn die politische Szene als Kampfarena von zwei politischen Lagern genutzt wird. Bei einem brisanten Redegefecht zwischen politischen Gegnern, so Bralczyk, werden gegenseitige Beschuldigungen eingesetzt, die eben auf diese beiden metaphorischen Felder zurückgreifen - Aggression (Krieg) und Lüge (Theater) (vgl. Bralczyk 2015). In der Diskussion um die Sprachverwendung im Raum der Politik wird nicht nur in jüngster Zeit nahezu durchgehend die Meinung von einer von Persuasion und Manipulation durchdrungenen Politikersprache sowie von zur Selbstdarstellung, Parteipropaganda und Diffamierung politischer Konkurrenz missbrauchten, inszenierten Scheingesprächen und Redekämpfen, rituellen Scheingefechten und Schaufensterparlamentarismus vertreten (vgl. Burkhardt 2003: 7 u. 1995; Kißler 1989: 1015; vgl. u. a. auch Tillmann 1989: 126; Holly 1990; Mikołajczyk 2004 sowie Kämper/Wengeler 2017). Bei Formen politischen Sprechens scheint es sich in vielen Fällen um persuasive bzw. propagandistische Textsorten zu handeln, bei denen Imagepflege sowie Diskreditierung politischer Konkurrenz und im Endeffekt Machterwerb bzw. Machterhalt als übergeordnetes Redeziel gelten (vgl. Tillmann 1989: 126). Die Auffassung des öffentlichen politischen Diskurses im Sinne der genannten Kriegsmetaphorik als etwa „Kampf ums Recht im Raum der Sprache“ (Frese 1972: 
105; vgl. Bergsdorf 1983: 293) oder „Kampf ums Heißen“ (Lübbe 1979: 53) bzw. „Kampf um Macht und Behauptung von Macht“ (Bergsdorf 1983: 27) oder „Kampf um Begriffe“ (vgl. Bergsdorf 1985) lässt unabdingbar ein bestimmtes Gewaltpotential vermuten, das sich in Form von allgemeinen Ausdrücken von Hass gegenüber dem/den Adressaten manifestiert. Aus dieser Perspektive werden an einigen Stellen auch die nicht-sprachlichen Faktoren im öffentlichen politischen Diskurs unterstrichen: die Sprache, ohne deren relevante Funktion zu leugnen, kann gewissermaßen als „Gewaltersatz" beschrieben werden (vgl. Holly 1990: 4). Solange die Sprache als das Medium politischer Auseinandersetzungen angenommen wird, so besteht nach Bergsdorf die Chance, auf andere Mittel wie zum Beispiel Gewalt zu verzichten (vgl. Bergsdorf 1983: 27).

Doch kann der Sprache selbst eine Handlungsmacht zugeschrieben werden, andere zu verletzen? Haben Wörter tatsächlich eine verwundende Macht? Und schließlich: scheitern Versuche der Begründung der Unbestreitbarkeit der verletzenden Macht anstößiger Äußerungen, wie Judith Butler in ihrem Haß spricht... plädiert (vgl. Butler 2016: 27), wirklich an der Frage danach, wer diese Äußerungen interpretiert, was diese Worte bedeuten, in welchen Kontexten und unter welchen institutionellen Bedingungen sie geäußert werden?

In der alltäglichen Auffassung wird Hassrede zunächst als eine aus dem englischen Hate Speech hergeleitete Bezeichnung für „menschenverachtend[e] Aussagen“ verstanden, in denen „Einzelne oder Gruppen abgewertet [werden]“" Hate Speech wird damit als „ein Oberbegriff für das Phänomen der gruppenbezogenen Menschenfeindlichkeit oder Volksverhetzung im Internet und Social-Media-Räumen" 2 betrachtet, der seinen Ausdruck „,in gewalttätiger Sprache findet" ${ }^{3}$. Zugleich wird auch auf „die fehlende Begriffsschärfe“ hingewiesen, sodass „dieser als politischer Begriff mit mehr oder weniger starken Bezügen zu juristischen Tatbeständen [gilt]"4. Als Muster von Hate Speech werden in diesem Zusammenhang etwa die bewusste Verbreitung uninformierter oder falscher Aussagen, herabwürdigende, verunglimpfende Begriffe und sexistische und rassistische Beleidigungen, Verallgemeinerungen, Wir/Die-Rhetorik, Verschwö-

\footnotetext{
${ }^{1}$ www.amadeu-antonio-stiftung.de/digitale-zivilgesellschaft/was-ist-hate-speech/ (01.05.2019).

${ }^{2}$ https://www.bpb.de/252396/was-ist-hate-speech (01.05.2019).

${ }^{3}$ www.amadeu-antonio-stiftung.de/digitale-zivilgesellschaft/was-ist-hate-speech/ (01.05.2019).

${ }^{4}$ https://www.bpb.de/252396/was-ist-hate-speech (01.05.2019).
} 
rungstheorien, plakative Bildsprache, Gleichsetzung, Befürwortung oder Androhung sexualisierter Gewalt oder Befürwortung von oder Aufruf zu Gewalttaten angeführt (vgl. AJS 2016: 12).

In der einschlägigen Literatur wird die Erscheinung von Hassrede nicht selten in der Konstellation mit anderen Phänomenen erörtert, wie etwa der Beleidigung (Meibauer 2013), Diffamierung (Kaczmarek 2018), Pejoration/ Pejorisierung (vgl. Finkeiner/Meibauer/Wiese 2016), sprachliche Gewalt (Klinker/Scharloth/Szczęk 2018) oder verbale Aggression (vgl. Bonacchi 2017), Hate und Haterei (poln. hejterstwo, vgl. Dynkowska et al. 2017) (Un)Höflichkeit (Bonacchi 2013), Populismus und Feindbildkommunikation (Niehr 2019; Niehr/Reissen-Kosch 2018; Pappert/Czachur 2019) oder Täuschung und Fake News (Antos 2017; Meibauer 2014). In der häufig angeführten Auffassung der Hate Speech nach Meibauer (2013) wird Hassrede als „der sprachliche Ausdruck von Hass gegen Personen oder Gruppen verstanden, insbesondere durch die Verwendung von Ausdrücken, die der Herabsetzung und Verunglimpfung von Bevölkerungsgruppen dienen" (Meibauer 2013: 1). Die Erscheinungsformen von Hassrede können dabei unterschiedlichste Gestalt annehmen - von offen, direkt, psychisch, physisch oder sozial verletzend bis getarnt, indirekt, latent, im theatralischen Sinn kostümiert und subliminal spürbar (vgl. Meibauer 2013: 1-3 u. Bonacchi 2017: 4-22). Beim sprachlichen Ausdruck von Hass können sprachliche und nicht-verbale Modalitäten miteinander kombiniert werden, es seien auch Faktoren zu berücksichtigen wie etwa Grad der Offenheit sowie die Unterstützung durch Autorität und Macht, Gewaltanteil, Intensität, Formen der Verschleierung (etwa durch Humor), Adressat(en) etc. (vgl. Meibauer 2013: 1-2). Bei seinem Systematisierungsversuch unter sprachwissenschaftlichem Aspekt verweist Meibauer (2013) auf die unterschiedlichsten Erscheinungsformen von Hassrede, wenn auch mit unterschiedlicher Intensität und Quantität, so doch „auf allen Ebenen des Sprachsystems und in der Sprachverwendung“. Hierzu gehören unter anderem prosodische Aspekte der Pejoration, pejorativierende Morpheme, Einsatz von Schimpfwörtern, pejorative Sprechakte, Strategien des Hassausdrucks in „Hasstexten“, und ggf. auch der Sprachwandel etwa beim Gewinn/Verlust beleidigender Kraft von Hate Speech-Ausdrücken oder Hate Speech-Sprechakten im Laufe der Zeit (vgl. Meibauer 2013: 3-7).

Aus politikwissenschaftlicher sowie rechtslinguistischer Sicht wird im Zusammenhang mit dem Phänomen von Hassrede auf die umstrittene Frage der Verträglichkeit von Regulierungen des Ausdrucks von Hass mit dem Problem der Einschränkung der Meinungs- und Versammlungsfreiheit 
(vgl. Meibauer 2013: 8-10; siehe auch Reissen-Kosch 2016: IX) hingewiesen. In diesem Zusammenhang verweist Reissen-Kosch etwa auf die Debatte um das NPD-Verbotsverfahren in Deutschland, zum einen als Beweis für die genannte Problematik der Verträglichkeit von Regelungen zum Schutz an Demokratie und Menschenwürde mit der grundgesetzlichen Redefreiheit, zum anderen aber zugleich als Beispiel dafür, dass etwa der Rechtsextremismus „trotz aller Aufklärungs- und Präventionsbemühungen in Deutschland nach wie vor präsent ist" (Reissen-Kosch 2016: IX; vgl. Wolf 2006). Im Kontext um das Engagement „in einen Kampf um die lexikalische Macht“, „die Deutungshoheit über die Begriffe, die uns benennen, zu übernehmen“ und damit „die Macht der Benennung für unsere eigenen Zwecke zurück[zu]gewinnen" (Butler 2016: 260) konstatiert Butler die alternative eines Redeverbots (wohl als zugespitzte Form von Counter Speech ${ }^{5}$ ) aus der Perspektive dessen Wirksamkeit:

Man sollte jedoch hinterfragen, inwiefern ein Redeverbot wirklich die Macht hat, die hate speech in ihren verletzenden Eigenschaften zu mindern bzw. ihr entgegenzuwirken. Es ist natürlich einerseits möglich, bestimmte Ausdrücke zu verbieten, und es gibt oft genug gute Gründe, dies auch zu tun. Allerdings kann das Verbot allein nicht bis zur Quelle des Hasses, die der hate speech mit ihren verletzenden Eigenschaften zugrunde liegt, vordringen. Wenn Wörter einfach verboten bleiben, dann wird ihre Bedeutung als fest eingefroren und bleibt nur als das Unausgesprochene und Unaussprechliche in Erinnerung. Um aber an die Wurzeln der hate speech zu gelangen, muß man darüber sprechen bzw. dagegen sprechen, und man muß Wege des Wieder-Sprechens finden, die dieser Rede ihre verletzende Wirkung entziehen. Sonst heiligt das Verbot die Verletzung erst und macht es letztlich sehr viel schwieriger zu verstehen, warum uns solche Verletzungen zugefügt werden und warum wir unter ihnen so leiden müssen. Wir sollten schließlich gründlich überdenken, wie wir eine Welt erschaffen können, in der unsere sprachliche Verwundbarkeit ausreichend geschützt ist (Butler 2016: 261).

Dem komplexen Phänomen des sprachlichen Ausdrucks von Hass wird im vorliegenden Band eine multidisziplinäre Herangehensweise sowie methodologische Vielfältigkeit entgegengebracht. Durch die Beteiligung deutscher und polnischer Forscher und Forscherinnen hat der Sammelband einen interdisziplinären und auch teils kontrastiven Ansatz. Der einleiten-

\footnotetext{
${ }^{5}$ Im Sinne einer aktiven Gegenrede und Reaktionsmöglichkeit auf den Ausdruck von Hass, vgl. AAS 2017: 4-5, siehe hierzu auch www.amadeu-antonio-stiftung.de/digitalezivilgesellschaft/was-ist-hate-speech/ (01.05.2019).
} 
de Beitrag Ethik der Sprache von Jerzy Bralczyk enthält Überlegungen zum aktuellen öffentlichen und politischen Sprachgebrauch in Polen. In den Vordergrund treten gewählte Erscheinungsformen von sprachlicher Gewalt, Hassrede sowie Sprachmanipulation und Sprachmissbrauch im öffentlichen Diskurs. Gegenstand des Beitrags von Jarosław Płuciennik und Michał Wróblewski Hassrede und Redefreiheit. Ein konzeptueller kulturwissenschaftlicher und anthropologischer Rahmen ist ein Überblick über die Erörterungen von jungen Forscher/innen zur Thematik, kritischen Strömungen sowie methodologischen Diskursen im Hinblick auf die Erscheinungen von Hassrede und Hate innerhalb einer vernetzten Gesellschaft. Die Interpretation der Forschungstendenzen innerhalb der humanistischen sowie soziopolitischen Wissenschaften wird mit besonderer Berücksichtigung der Entwicklung der Ideen von Redefreiheit präsentiert. Der anschließende Beitrag Fiktive Feindbilder und verschleierte Gewalt. Sprachwissenschaftliche Analysen zum Interview mit einem Islamisten von Albrecht Greule, Sandra Reimann und Anna Schuster untersucht ein Interview mit einem „Bekenner“ des Islamischen Staates, welches im Magazin der Süddeutschen Zeitung erschienen ist. Dabei sollen über Auffälligkeiten in der Wortwahl islamistische Denkmuster herausgearbeitet werden, den Fokus bei der Analyse bildet die Versprachlichung von Kollektivbezeichnungen und verschleierter Gewalt. Im folgenden Beitrag Feindbildkommunikation. Polarisieren und Diskreditieren im politischen Diskurs befasst sich Heinz-Helmut Lüger mit dem Einsatz von Feindbildern, die sprachlich etwa durch das Formulieren negativer Bewertungen aber auch Manifestation von Verachtung oder Hass realisiert werden können. Die Erörterungen und Belege betreffen den historischen Sachverhalt des Ausbruchs des Ersten Weltkrieges. Vor dem Hintergrund des Phänomens der Hassrede und der Diskurskritik präsentieren Waldemar Czachur und Marta Smykała in ihrem Beitrag Hassrede und Diskurskritik. Kontrastive Analyse der Mediendiskurse über Migration in Polen und in Deutschland zu Beginn der Flüchtlingskrise in Europa die Ergebnisse einer kontrastiven Analyse des Mediendiskurses in Polen und Deutschland zu Beginn der sogenannten Flüchtlingskrise. Den Schwerpunkt der Untersuchung bilden die Diskurse in den Zeitschriften Wprost, Gazeta Polska, Polityka und Die Zeit. Der anschließende Beitrag von Monika Kopytowska und Agnieszka Stawikowska-Marcinkowska »Der Fremde«. Flüchtlingskrise und Hassrede in der Online-Version widmet sich dem Phänomen von Hassrede im Internet gegenüber dem „Fremden“ in Polen und Deutschland. Die Autorinnen versuchen die Frage nach der eigent- 
lichen Identität „des Fremden“ zu beantworten, welcher oft zum Gegenstand von Stereotypen, Vorurteilen und zuletzt verbaler Aggression wird. Während die Autorinnen auf den universellen Charakter der gewählten Mittel und sprachlichen Strategien innerhalb der gegenüber den genannten Personengruppen gerichteten Hassrede eingehen, thematisieren sie zugleich den Aspekt historischer, rechtlicher sowie sozioökonomischer Bedingungen in den beiden besprochenen Ländern. Der Beitrag Hostis honori invidia - Hass macht dem Gegner Ehre. Fremdenhass und Aussöhnung. Eine exemplarische Analyse unter dem Aspekt des historischen »Deutschenhasses « und dessen Überwindung in der Relation zwischen Deutschen und Polen von Witold Sadziński legt den Hauptakzent auf den Prozess der Aussöhnung zwischen Deutschen und Polen. Dies geschieht aus der Überzeugung heraus, Fremdenhass sei meist das Resultat mangelnder Kommunikation. Der folgende Beitrag von Lukasz M. Plęs zum VRP-Neusprech im Spiegel der Aufzeichnungen von Michat Gtowiński befasst sich mit der Problematik des Neusprechs in der Volksrepublik Polen unter besonderer Berücksichtigung der Kommentare von Michał Głowiński. Als Ausdruck scharfer Kritik und tiefgründiger Analyse der Propagandasprache der Staatsgewalt stützten sich die Kommentare vor allem auf die Lektüre der Tageszeitung Trybuna Ludu. Roman Opiłowski befasst sich in seinem Beitrag Netzhass in deutschen und polnischen Nutzerkommentaren aus multimodaler Sicht mit dem Thema Hassrede als symbolischer Gewalt und gleichzeitig der realen sprachlichen Gewalt am Beispiel von Nutzerkommentaren zum Terroranschlag in Nizza im Juli 2016. Mit einer kontrastierenden und multimodalen Methode zur Analyse deutscher und polnischer Kommentare werden Themenfelder definiert, gefolgt von sprachlichen und multimodalen Handlungen, die den zwischenmenschlichen Hass zum Ausdruck bringen. Ziel des anschließenden Beitrags Abkehr vom Frieden? Eine medien- und politolinguistische Untersuchung von Facebook-Einträgen der Organisation Pegida von Albrecht Greule, Sandra Reimann und Julia Enzinger ist es aufzuzeigen, was den Sprachgebrauch Pegidas im Rahmen von Facebook-Einträgen kennzeichnet und wie Sprache eingesetzt wird, um auf oder über das soziale Netzwerk Zustimmung zu erreichen. Dabei wird untersucht, inwiefern der Verein Sprache als Instrument zur Anhängerwerbung einsetzt und ob Pegida Sprache tatsächlich als Mittel zur Kritik an Missständen und Gegnern verwendet. Im Beitrag Von Imagepflege zur Diskreditierung politischer Gegner. Hassrede als Strategie von Online-Pressemitteilungen von Jacek Makowski richtet sich die wesentliche Fragestellung nach pejorativierenden, diskreditierenden und abwertenden 
Strategien sowie dem Einsatz von verschiedenen Erscheinungsformen von Hassrede in der Textsorte Online-Pressemitteilung. Auf das Untersuchungskorpus setzt sich dabei der offiziellen Webseite der Alternative für Deutschland (AfD) entnommenes Textmaterial zusammen, welches in Anlehnung an die erarbeiteten Erkenntnisse zur Pressemitteilung als politischer Textsorte einer textsortenorientierten Analyse unterzogen wird.

\section{Literaturverzeichnis}

Amadeu Antonio, Stiftung (2017) (AAS 2017): Toxische Narrative. Monitoring rechtsalternativer Akteure. Berlin.

Antos, Gerd (2017): Fake News. Warum wir auf sie reinfallen. Oder: »Ich mache euch die Welt, so wie sie mir gefällt«. In: Der Sprachdienst 1/2017, S. 1-20.

Arbeitsgemeinschaft Kinder und Jugendschutz et al. (2016) (AJS 2016): Hate Speech - Hass im Netz. Köln.

Bergsdorf, Wolfgang (1983): Herrschaft und Sprache. Studie zur politischen Terminologie der Bundesrepublik Deutschland. Pfullingen.

Bergsdorf, Wolfgang (1985): Kampf um Begriffe. Aufgaben der politischen Semantik. In: Universitas 40, S. 1245-1252.

Bonacchi, Silvia (Hg.) (2017): Verbale Aggression. Multidisziplinäre Zugänge zur verletzenden Macht der Sprache. Berlin.

Bralczyk, Jerzy (2016): Etyka języka. In: Academia 1(45), S. 57.

Burkhardt, Armin (1995): Zwischen Diskussions- und Schaufensterparlamentarismus. Zur Diagnose und Kritik parlamentarischer Kommunikation - am Beispiel von Zwischenfragen und Kurzdialogen. In: Dörner, Andreas/Vogt, Ludgera (Hg.): Sprache des Parlaments und Semiotik der Demokratie. Studien zur politischen Kommunikation in der Moderne. Berlin/New York, S. 73-106.

Burkhardt, Armin (2003): Das Parlament und seine Sprache. Studien zu Theorie und Geschichte parlamentarischer Kommunikation. Tübingen.

Butler, Judith (2016): Haß spricht. Zur Politik des Performativen. 5. Auflage. Berlin.

Dieckmann, Walther (1975): Sprache in der Politik. Einführung in die Pragmatik und Semantik der politischen Sprache. Heidelberg.

Dieckmann, Walther (1983): Sprache und Kommunikation in politischen Institutionen. In: Linguistische Arbeiten und Berichte. Hg. v. Fachbereich 16 der FU Berlin, S. 1-66.

Dynkowska, Julia et al. (2017): Hejterstwo. Nowa praktyka kulturowa? Łódź.

Finkeiner, Rita/Meibauer, Jörg/Wiese, Heike (Hg.) (2016): Pejoration. Amsterdam/ Philadelphia.

Frese, Jürgen (1972): Politisches Sprechen. Thesen über einige Rahmenbedingungen. In: Rucktäschel, Annamaria (Hg.): Sprache und Gesellschaft. München, S. $102-114$. 
Holly, Werner (1990): Politikersprache. Inszenierungen und Rollenkonflikte im informellen Sprachhandeln eines Bundestagsabgeordneten. Berlin.

Kaczmarek, Dorota (2018): Binationale Diskursanalyse. Grundlagen und Fallstudien zum deutsch-polnischen medialen Gegendiskurs. Łódź.

Kämper, Heidrun/Wengeler, Martin (Hg.) (2017): Protest - Parteienschelte - Politikverdrossenheit: Politikkritik in der Demokratie. Bremen.

Kißler, Leo (1989): Parlamentsöffentlichkeit: Transparenz und Artikulation. In: Schneider, Hans-Peter/Zeh, Wolfgang (Hg.): Parlamentsrecht und Parlamentspraxis in der Bundesrepublik Deutschland. Berlin/New York, S. 993-1020.

Klinker, Fabian/Scharloth, Joachim/Szczęk, Joanna (Hg.) (2018): Sprachliche Gewalt. Formen und Effekte von Pejorisierung, verbaler Aggression und Hassrede. Stuttgart.

Lübbe, Hermann (1979): Sein und Heißen - Bedeutungsgeschichte als politisches Sprachhandlungsfeld. In: Bergsdorf, Wolfgang (Hg.): Wörter als Waffen. Sprache als Mittel der Politik. Stuttgart, S. 71-84.

Meibauer, Jörg (2013): Hassrede - von der Sprache zur Politik. In: Meibauer, Jörg (Hg.): Hassrede/Hate Speech. Interdisziplinäre Beiträge zu einer aktuellen Diskussion. Gießener elektronische Bibliothek. Gießen, S. 1-16. Quelle: http://geb.uni-giessen.de/geb/volltexte/2013/9251 (22.12.2018).

Meibauer, Jörg (2014): Lying at the Semantivs-Pragmatics Interface. Boston/Berlin. Mikołajczyk, Beata (2004): Sprachliche Mechanismen der Persuasion in der politi-schen Kommunikation. Dargestellt an polnischen und deutschen Texten zum EU-Beitritt Polens. Frankfurt am Main.

Niehr, Thomas (2019): Populismus - der Extremismus von heute? In: Sprachreport 1, S. 24-30.

Niehr, Thomas/Reissen-Kosch, Jana (2018): Volkes Stimme? Zur Sprache des Rechtspopulismus. Berlin.

Pappert, Steffen/Czachur, Waldemar (2019): Visueller Populismus: Eine Analyse multimodaler Praktiken anhand von Wahlplakaten aus Deutschland und Polen. In: Osnabrücker Beiträge zur Sprachtheorie 95/2019, S. 103-127.

Reissen-Kosch, Jana (2016): Identifikationsangebote der rechten Szene im Netz. Linguistische Analyse persuasiver Online-Kommunikation. Bremen.

Silvia, Bonacchi (2013): (Un)höflichkeit. Eine kulturologische Analyse Deutsch - Italienisch - Polnisch. Frankfurt am Main.

Tillmann, Alexander (1989): Ausgewählte Textsorten politischer Sprache. Eine linguistische Analyse parteilichen Sprechens. Göppingen.

Wolf, Joachim (2006): Die NPD-Verbotsdebatte. Quelle: www.bpb.de/politik/extremismus/rechtsextremismus/41872/debatte-ums-npd-verbot (01.05.2019). 